\title{
Research on Several Problems Related to Immigrant
}

\author{
Ruijie Zhang $^{1}$ \\ ${ }^{1}$ School of North China Electric Power University, Hebei 071000, China; \\ a646419481@qq.com
}

Keywords: Monte Carlo method, analytic hierarchy process, grey relational analysis

\begin{abstract}
We define parameters related to income, education and social equality, and choose a certain number of sample members. Monte Carlo method is applied based on the census dataset. Combining analytic hierarchy process and grey relational analysis, we construct the model to evaluate Population Zero and the indicator of Population Zero from grey relation grades of the sample members.

Divide the workforce into the professional and the unskilled. We introduce different coefficients for different group and factor, then we construct a model which can reflect influence to people in terms of each factor, and define each groups' priorities. We build a model to evaluate how closely their needs are met in terms of the three factors concerned with the urban development and happiness, while a higher indicator represents a high level of happiness. Considering the proportion of the professional and unskilled workforce as well as the corresponding influence to people, we construct the global optimized model including different subgroups.
\end{abstract}

\section{Introduction}

The international agency, Laboratory of Interstellar Financial \& Exploration Policy (LIFE), has recently (in this year of 2095) completed a series of short-term planned living experiments on our neighbor planet, Mars. New technologies will soon enable humans to inhabit manufactured cities on Mars by 2100. The first wave of migration, called Population Zero, will include 10,000 people.

The LIFE agency launched project UTOPIA: 2100, with the goal of creating an optimal workforce for the 22nd century to give all people the greatest quality of life with a vision of sustainability for the next 100 years.

We establish the hierarchical evaluation model for Population Zero with a three-layer system. Use grey relation analysis to get grey relation grades of the sample members and construct indicator of Population Zero. Based on the total data for all the sample members obtained by Monte Carlo method, we can get the result of the indicator.

In the model to evaluate priority for different subgroup, we neglect the social part and only consider the influence to people in terms of the three factors. Construct models to evaluate influence to people in terms of each factor respectively and introduce different coefficients for each factor when considering the total influence to people. Product of the quantized and corresponding coefficient reflects the weight of each in the influence to people.

Since both the indicator of sustainable urban development and influence to people are relative to the three factors, satisfaction to evaluate how their needs are met in terms of the three factors can be described as an expression concerned with indicator of sustainable urban development and influence to people.

Considering the proportion of the professional and unskilled workforce as well as the corresponding influence to people, we construct the optimized model to evaluate the needs of the two different subgroups. 


\section{Indicator of Population Zero}

\section{Data for Sample Members by Monte Carlo Method.}

To study the three priority factors (income, education, and social equality), we enrich the intension and make some extensions. Features of the population like gender, age, academic qualification and job category are taken into consideration, while job category refer to the professional workforce or unskilled workforce.

Several metrics are identified and defined for each of the three factors shown in Figure 1. Specifically, job category and wage/salary serve as metrics to evaluate the income, while academic qualification, expenditure on skills training and expenditure on childcare for the education; and gender, age, maternity and paternity leave for the social equality.

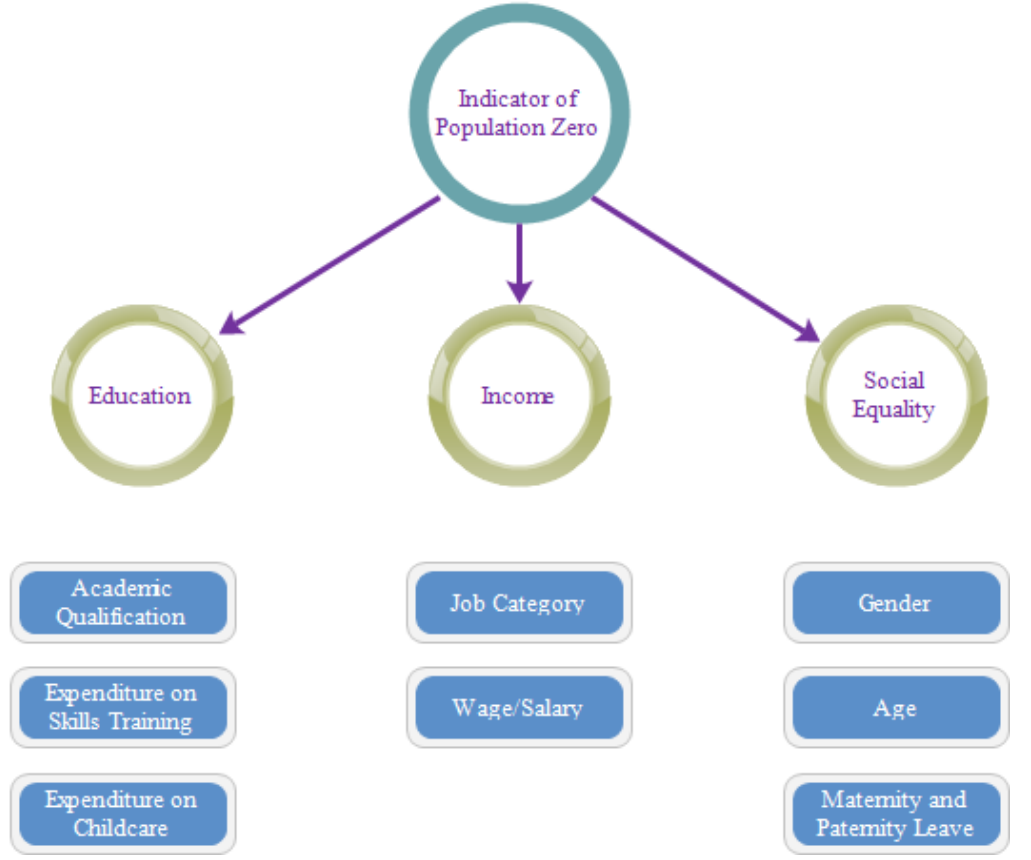

Figure1. Hierarchical evaluation model for indicator of Population Zero

A certain number of members are chosen in our sample to reflect the Population Zero, and Monte Carlo method is applied to establish the various estimators. For metrics with random properties like job category, wage/salary, academic qualification and gender, we use Monte Carlo simulation and generate random numbers for members of the sample. Data distribution of these metrics is shown in Figure 2. For definite metrics without random properties like expenditure on skills training, expenditure on childcare, maternity and paternity leave, based on the census dataset, we extract probabilities of them and generate random numbers with the known probability distribution. In this way, we can get total data of metrics for the three factors for all the sample members.

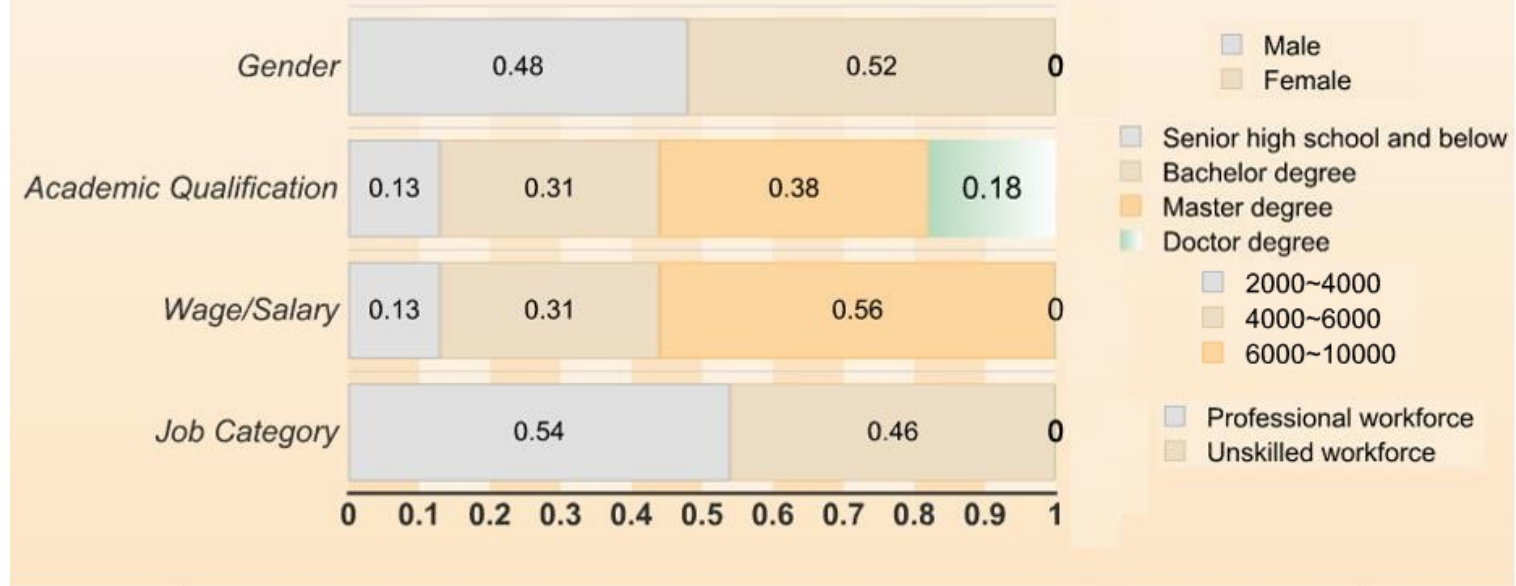

Figure2. Data distribution of metrics with random properties 


\section{Indicator of Population Zero by Grey Relational Analysis.}

The hierarchical evaluation model for Population Zero consists of a three-layer evaluation system shown in Figure 1. [1]The first layer is the basic layer composed of the specific metrics, and their mutual combinations constitute the second layer of the three factors, while the third layer of the indicator of Population Zero is determined by the three factors in the second layer.

Next, we use the grey relational analysis and get grey relation grades of the sample members, therefore, the indicator of Population Zero can be constructed.[2]

With sample members as the evaluation objects and the total eight metrics as the evaluation indexes, the reference sequence is $x_{0}=\left\{x_{0}(k) \mid k=1,2, \ldots, 8\right\}$, the comparison sequence is $x_{i}=\left\{x_{i}(k) \mid k=1,2, \ldots, 8\right\}, \mathrm{i}=1,2, \ldots, \mathrm{m}$, where $\mathrm{m}$ is the number of our sample members. We consider the corresponding weight of each index to be equal, that is $1 / 8$. Grey relation coefficient $\xi_{i}$ is the relation coefficient of metric $k$ between the comparison sequence $x_{i}$ and the reference sequence $x_{0}$, which can be calculated as

$$
\xi_{i}(k)=\frac{\min _{s} \min _{t}\left|x_{0}(t)-x_{s}(t)\right|+\rho \max _{s} \max _{t}\left|x_{0}(t)-x_{s}(t)\right|}{\left|x_{0}(k)-x_{i}(k)\right|+\rho \max _{s} \max _{t}\left|x_{0}(t)-x_{s}(t)\right|} .
$$

Among the formula, resolution coefficient $\rho \in[0,1]$, which is usually valued 0.5 , and we consider $\rho=0.5$. Need to point out thatmin $\min _{t}\left|x_{0}(t)-x_{s}(t)\right|$ and $\max _{s} \max _{t}\left|x_{0}(t)-x_{s}(t)\right|$ are minimum and maximum differences $\Delta_{\min }$ and $\Delta_{\max }$ respectively.

The grey relation grade of evaluation object irelative to the ideal object $r_{i}$ is

$$
r_{i}=\frac{1}{8} \sum_{k=1}^{8} \xi_{i}(k) \text {. }
$$

Thus, grey relation grade $r_{i}$ for all the sample members can be obtained. We calculate the expectation and variance of all the grey relation grade $r_{i}$ and consider them as two sub-indicators $\eta_{1}$ and $\eta_{2}$, which can be described as

$$
\begin{aligned}
& \eta_{1}=E(\mathrm{x})=\frac{1}{m} \sum_{i=1}^{m} r_{i}, \\
& \eta_{2}=D(x)=\mathrm{E}\left\{[x-E(x)]^{2}\right\} .
\end{aligned}
$$

\section{Model to Evaluate Priority for Different Subgroup}

We divide the workforce into two subgroups: the professional workforce and unskilled workforce. Since their priorities are different, we need to build a model to evaluate their main priorities.

Based on the revised minimum wage $\frac{\min }{m w s}=\frac{e^{\alpha_{1} \ln u+\alpha_{2} \ln w}}{m w s},{ }^{[3]}$ we change the social welfare to the working hours $h$ when it comes to influence to people. Therefore, the influence to people in terms of income $t_{1}{ }^{\prime}$ can be described as

$$
t_{1}^{\prime}=\frac{e^{\alpha_{1} / \ln u+\alpha_{2} / \ln h}}{m w s} .
$$

With a high level of unemployment rate as well as the working hours and a low minimum wage standard, people will be affected seriously by income and we think influence of income reflects a positive impact to people.

Influence to people in terms of education just considers the two priorities for different subgroups, and take the linear addition of training expenditure as well as the child care expenditure. In correspondence, we divide the sum of training expenditure $e$ and child care expenditure ce by the minimum wage standard $m w s$ of the same dimension. Influence to people in terms of education $t_{2}{ }^{\prime}$ can be described as

$$
t_{2}^{\prime}=\frac{\text { training expenditure }+ \text { child care expenditure }}{\text { minimum wage standard }}=\frac{t e+c e}{m w s} .
$$

While higher training expenditure and child care expenditure reflect a higher level of the living standard for the citizens, we think the influence to people in terms of education $t_{2}$ plays a positive effect.

Take the linear addition of normal holiday $n h$ and maternity, paternity leave $m p$, we get the total time off for people, particularly, take $m p=0$ without newborn baby.In correspondence, we divide 
the total time off ( $n h+m p)$ by 365,the days of a year, of the same dimension.Influence to people in terms of social equality $t_{3}{ }^{\prime}$ can be described as

$$
t_{3}^{\prime}=\frac{n h+m p}{365} \text {. }
$$

With a longer normal holiday and maternity, paternity leave, people will be affected seriously by social equality. Generally, people will be more satisfied by a longer holiday, therefore, we think influence of social equality reflects a positive impact to people.

To evaluate the priority for different subgroups, influence of the three identified factors (income, education and social equality) to people $t_{p}$ is no longer the simple linear addition of influence of each factor to people $t_{1}{ }^{\prime}, t_{2}{ }^{\prime}, t_{3}$, different coefficients $\beta_{1}, \beta_{2}, \beta_{3}$ are introduced for each factor in the total influence to people. Product of influence of each factor to people and the corresponding coefficient represents the weight of each factor in the total influence to people. Influence of the three identified factors to people $t_{p}$ can be described as

$$
t_{p}=\beta_{1} t_{1}^{\prime}+\beta_{2} t_{2}^{\prime}+\beta_{3} t_{3}^{\prime}=\beta_{1} \frac{e^{\alpha_{1} \ln u+\alpha_{2} / \ln h}}{m w s}+\beta_{2} \frac{t e+c e}{m w s}+\beta_{3} \frac{n h+m p}{365} .
$$

\section{Conclusions}

In the model of indicator of Population Zero, the sub-indicator $\eta_{1}$ reflects the general development level of Population Zero, while the sub-indicator $\eta_{2}$ reflects the degree of dispersion of the development level. The two sub-indicators $\eta_{1}$ and $\eta_{2}$ form the indicator of Population Zero $\eta$. Based on the total data for all the sample members obtained by Monte Carlo method, we get results of the two sub-indicators and $\eta_{1}=0.9167, \eta_{2}=0.0012$.

In the model to evaluate priority for different subgroup, product of $t_{i}{ }^{\prime}$ and corresponding coefficient $\beta_{i}(\mathrm{i}=1,2,3)$ reflects the weight of factor $\mathrm{i}$ in influence to people. That is to say, a higher $\beta_{1} t_{1}^{\prime}$ means people are more likely to be affected by income, a higher $\beta_{2} t_{2}^{\prime}$ means people are more likely to be affected by education and a higher $\beta_{3} t_{3}^{\prime}$ means people are more likely to be affected by social equality. For different group, we assign different value to the coefficients $\beta_{1}, \beta_{2}$, $\beta_{3}$, while influence of each factor to people $t_{1}{ }^{\prime}, t_{2}{ }^{\prime}, t_{3}$ 'depend on the realistic magnitude of different subgroup. Therefore, we are able to identify the priority of the three factors for different subgroups.

\section{Reference}

[1] Mordeson J N, Wierman M J, Clark T D, et al. The Analytic Hierarchy Process[M]// Linear Models in the Mathematics of Uncertainty. 2013:19-28.

[2] Yi H F. Study on Comprehensive Evaluation Model for Combined Cooling Heating and Power System (CCHP)[J]. Journal of Engineering Thermophysics, 2005.

[3] Ning G J. Chinese Minimum Wage Standard Formulation and Adjustment According to the Empirical Analysis[J]. Chinese Population Science, 2011, 2011 (1): 26-34. 